\title{
Roles of Adiponectin and Oxidative Stress in the Regulation of Membrane Microviscosity of Red Blood Cells in Hypertensive Men-An Electron Spin Resonance Study
}

\author{
Kazushi Tsuda ${ }^{1,2}$ \\ ${ }^{1}$ Cardiovascular and Metabolic Research Center, Kansai University of Health Sciences, Senn-nann-gunn, Kumatori-cho, \\ Wakaba 2-11-1, Osaka 590-0482, Japan \\ ${ }^{2}$ Division of Cardiology, Department of Medicine, Wakayama Medical University, Wakayama 641-8509, Japan
}

Correspondence should be addressed to Kazushi Tsuda, tsudak@mail.wakayama-med.ac.jp

Received 27 May 2010; Accepted 18 July 2010

Academic Editor: S. B. Heymsfield

Copyright ( $) 2011$ Kazushi Tsuda. This is an open access article distributed under the Creative Commons Attribution License, which permits unrestricted use, distribution, and reproduction in any medium, provided the original work is properly cited.

\begin{abstract}
This study was undertaken to investigate possible relationships among plasma adiponectin, 8-iso-prostaglandin F2 $\alpha$ (8-iso-PG F $2 \alpha$ : an index of oxidative stress), and membrane fluidity (a reciprocal value of microviscosity) in hypertensive and normotensive men using an electron spin resonance-method. The order parameter (S) for the spin-label agent (5-nitroxide stearate) in red blood cell (RBC) membranes was higher in hypertensive men than in normotensive men, indicating that membrane fluidity was decreased in hypertension. Plasma adiponectin and NO metabolites levels were lower in hypertensive men than in normotensive men. In contrast, plasma 8-iso-PG F2 $\alpha$ levels were increased in hypertensive men compared with normotensive men. Plasma adiponectin concentration was correlated with plasma NO-metabolites, and inversely correlated with plasma 8-iso-PG F2 $\alpha$. The order parameter (S) of RBCs was inversely correlated with plasma adiponectin and plasma NO metabolite levels, and positively correlated with plasma 8-iso-PG F2 $\alpha$, suggesting that the reduced membrane fluidity of RBCs might be associated with hypoadiponectinemia, endothelial dysfunction, and increased oxidative stress. In a multivariate regression analysis, adiponectin and 8-iso-PG F2 $\alpha$ were significant determinants of membrane fluidity of RBCs after adjustment for general risk factors. These results suggest that adiponectin and oxidative stress might have a close correlation with rheologic behavior and microcirculation in hypertension.
\end{abstract}

\section{Introduction}

It has been shown that dysregulation of adipocytokines may be accompanied by obesity, diabetes mellitus, dyslipidemia, and hypertension, and finally result in atherosclerotic vascular diseases [1-4]. Recently, it is strongly suggested that adiponectin, the most abundant secretory protein of adipose tissue in human plasma, might actively participate in the regulation of cardiovascular functions in humans, because hypoadiponectinemia might be observed in subjects with hypertension and other cardiovascular diseases [1-4]. It has been demonstrated that plasma adiponectin levels increased during weight reduction or blockade of the reninangiotensin system [5], indicating that adiponectin might be beneficial for preventing the development of atherosclerotic changes.
On the other hand, it has also been shown that oxidative stress might be involved in the pathophysiology of obesity, hypertension, and atherosclerosis, and might be associated with increased risk of cardiovascular diseases, vascular dysfunction, and the metabolic syndrome [6-8]. Evidence indicates that plasma 8-iso-prostaglandin F2 $\alpha$ (8-iso-PG F2 $\alpha$ ) may be a reliable index of oxidative stress in humans [912]. It was demonstrated that plasma concentration of 8-isoPG F2 $\alpha$ was significantly increased in subjects with essential hypertension compared with normotensive subjects $[9,10]$. It was shown that plasma 8 -iso-PG F2 $\alpha$ levels were elevated in patients with coronary artery disease, particularly in those with hypertension [11, 12]. Moreover, it was shown that oral administration of vitamin E significantly decreased 8iso-PG F2 $\alpha$ concentrations in overweight/obese individuals, 
suggesting that a decrease in plasma 8 -iso-PG F2 $\alpha$ has the potential to reduce the risk of cardiovascular disease in obesity [13].

Many studies have focused on the cardioprotective effects attributable to nitric oxide (NO) and have shown that hypertension and other circulatory disorders may be associated with insufficient NO production and availability $[14,15]$. Chen et al. [16] demonstrated that adiponectin may stimulate production of NO in vascular endothelial cells. It has been shown that plasma adiponectin was correlated with endothelium-dependent vasodilation of the brachial artery in humans $[2,17]$. In contrast, it was shown that endothelium-dependent vasodilation was impaired in subjects with elevated oxidative stress levels $[18,19]$. These findings suggest that adiponectin and 8 -iso-PG F2 $\alpha$ might have a role in the production and bioavailability of NO.

It has been proposed that abnormalities in physicochemical properties of the cell membranes may underlie the defects that are strongly linked to hypertension, stroke, and other cardiovascular diseases [20-22]. An electron spin resonance (ESR) and spin-labeling method has been developed to evaluate the membrane fluidity and perturbations of the membrane function by external agents $[21,22]$. The membrane fluidity is a reciprocal value of membrane microviscosity and is an important factor in modulating the cell rheological behavior $[21,22]$. We have shown that the membrane fluidity of red blood cells (RBCs) was significantly lower in both spontaneously hypertensive rats (SHR) and patients with essential hypertension than in the normotensive controls [23-26], and proposed that abnormal membrane fluidity of RBCs might contribute to the pathogenesis of hypertension. In a study presented recently, we showed that adiponectin alone [27] or 8-iso-PG F2 $\alpha$ alone [28] might be determinants of membrane fluidity of RBCs. In addition, it has been demonstrated that NO may be involved in the regulation of cell membrane fluidity [29]. Our previous in vitro study showed that an NO donor significantly improved membrane fluidity of RBCs in subjects with essential hypertension [30], indicating that $\mathrm{NO}$ could have a beneficial effect on the rheologic behavior of RBCs and the microcirculation in hypertension. The present study was performed to assess the relationships among adiponectin, oxidative stress, and NO, and their roles in the regulation of membrane fluidity of RBCs in hypertensive men using the ESR and spin-labeling method.

\section{Subjects and Methods}

2.1. Subjects. A total of 26 men with untreated essential hypertension were studied and compared with 17 agematched normotensive men. The characteristics and laboratory findings in both groups were shown in Table 1. All subjects had no history of haematologic or hepatic disorders. All men were nonsmokers. They had similar life styles and dietary habits, and were instructed to avoid any changes in dietary habits at least 12 weeks before the study. The study was approved by a local research committee of Kansai University of Health Sciences. Written informed consent

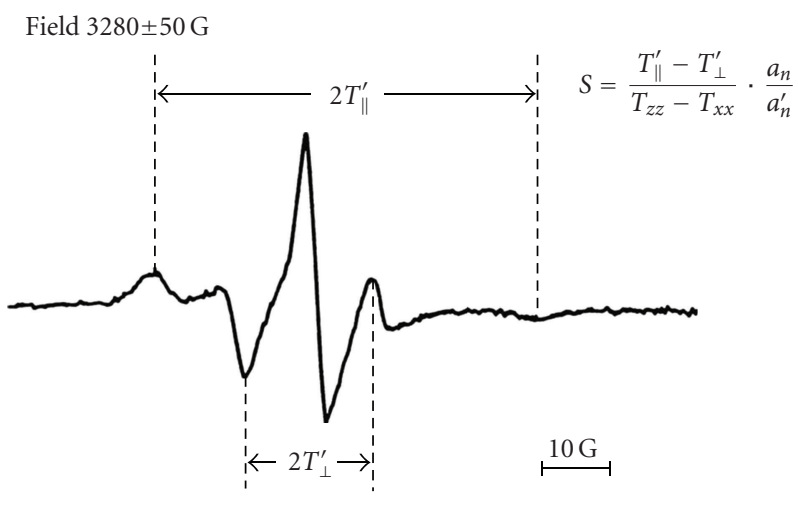

FIGURE 1: Representative electron spin resonance spectrum of red blood cells (RBCs) for the fatty acid spin-label agent (5-nitroxide stearate). $S$ : order parameter, $2 T_{\|}^{\prime}$ : outer hyperfine splitting, $2 T_{\perp}^{\prime}$ : inner hyperfine splitting, $T_{z z}$ and $T_{x x}$ : hyperfine constants, $a_{n} / a_{n}^{\prime}$ : isotropic coupling constant, G: gauss. The greater the value of the order parameter $(S)$ was, the lower the membrane fluidity of RBCs was.

was obtained from all participants after they were informed about the nature and objective of the study.

2.2. Measures. Blood sampling was performed by venipuncture after a 30 minutes of bed rest while fasting. The procedures of RBC preparation and ESR measurements were shown previously [22-27]. We evaluated the values of outer and inner hyperfine splitting $\left(2 \mathrm{~T}_{\|}^{\prime}\right.$ and $2 \mathrm{~T}_{\perp}^{\prime}$ in $\mathrm{G}$, resp.) in the ESR spectra for the spin label agent (5-nitroxide stearate, Aldrich Co. Ltd., Milwaukee, Wisconsin, USA) and calculated the order parameter $(S)$ [23-28] (Figure 1). The greater the value of the order parameter $(S)$ was, the lower was the membrane fluidity of RBCs [22-27].

Plasma 8-iso-PG F2 $\alpha$ levels were determined by using an enzyme immunoassay (Cayman Chemicals Co., Ann Arbor, Michigan, USA) $[10,11]$. The plasma levels of adiponectin and NO-metabolites (nitrite and nitrate) were measured according to the method described previously [1, 27, 28, 31].

2.3. Statistical Analysis. Values are expressed as mean \pm SEM. The differences between hypertensive and normotensive men were analyzed using an unpaired Student's $t$-test. Linear regression analysis was performed to assess the relationships among membrane fluidity (order parameter; $S$ ) of RBCs, plasma adiponectin, plasma 8 -iso-PG F $2 \alpha$, and plasma NOmetabolite levels. Multivariate regression analysis with membrane fluidity (order parameter; $S$ ) of RBCs as a dependent variable and plasma adiponectin, plasma 8 -iso-PG F2 $\alpha$, age, body mass index (BMI), fasting plasma glucose, systolic blood pressure, and plasma total cholesterol as independent variables was also performed. A $P$ value less than .05 was accepted as the level of significance.

\section{Results}

3.1. Membrane Fluidity of RBCs in Hypertensive and Normotensive Men. The order parameter $(S)$ for 5-nitroxide 
TABle 1: Clinical Characteristics and Laboratory Findings of Hypertensive (HT) and Normotensive (NT) Men.

\begin{tabular}{lcc}
\hline & & NT \\
\hline Number of subjects & 17 & HT \\
Age $($ y.o. $)$ & $65 \pm 3$ & $63 \pm 2$ \\
Body mass index $\left(\mathrm{kg} / \mathrm{m}^{2}\right)$ & $24.5 \pm 0.6$ & $24.1 \pm 0.6$ \\
Systolic blood pressure $(\mathrm{mmHg})$ & $124 \pm 2$ & $147 \pm 1^{*}$ \\
Diastolic blood pressure $(\mathrm{mmHg})$ & $69 \pm 2$ & $87 \pm 1^{*}$ \\
Heart rate $($ beats/min) & $75 \pm 1$ & $73 \pm 1$ \\
Erythrocyte counts $\left(10^{4}\right.$ cells/ $\mu \mathrm{L}$ & $458 \pm 11$ & $475 \pm 9$ \\
Hemoglobin $(\mathrm{g} / \mathrm{dL})$ & $14.1 \pm 0.3$ & $14.1 \pm 0.2$ \\
Hematocrit $(\%)$ & $43.1 \pm 1.0$ & $43.0 \pm 0.6$ \\
Leucocyte counts $\left(10^{3}\right.$ cells/ $\left.\mu \mathrm{L}\right)$ & $5.5 \pm 0.3$ & $5.4 \pm 0.2$ \\
Platelets $\left(10^{4}\right.$ cells/ $\left.\mu \mathrm{L}\right)$ & $20 \pm 1$ & $22 \pm 1$ \\
Total cholesterol $(\mathrm{mg} / \mathrm{dL})$ & $203 \pm 7$ & $210 \pm 7$ \\
High density lipoprotein cholesterol $(\mathrm{mg} / \mathrm{dL})$ & $50 \pm 3$ & $53 \pm 4$ \\
Low density lipoprotein cholesterol $(\mathrm{mg} / \mathrm{dL})$ & $127 \pm 6$ & $126 \pm 6$ \\
Triglycerides $(\mathrm{mg} / \mathrm{dL})$ & $137 \pm 18$ & $143 \pm 15$ \\
Serum sodium $(\mathrm{mmol} / \mathrm{L})$ & $140.7 \pm 0.4$ & $140.1 \pm 0.3$ \\
Serum potassium $(\mathrm{mmol} / \mathrm{L})$ & $4.1 \pm 0.1$ & $4.0 \pm 0.1$ \\
Serum creatinine $(\mathrm{mg} / \mathrm{dL})$ & $0.8 \pm 0.1$ & $0.9 \pm 0.1$ \\
Fasting plasma glucose $(\mathrm{mg} / \mathrm{dL})$ & $120 \pm 10$ & $119 \pm 9$ \\
\hline
\end{tabular}

Values are means \pm SEM. ${ }^{*} P<.05$ between HT and NT.

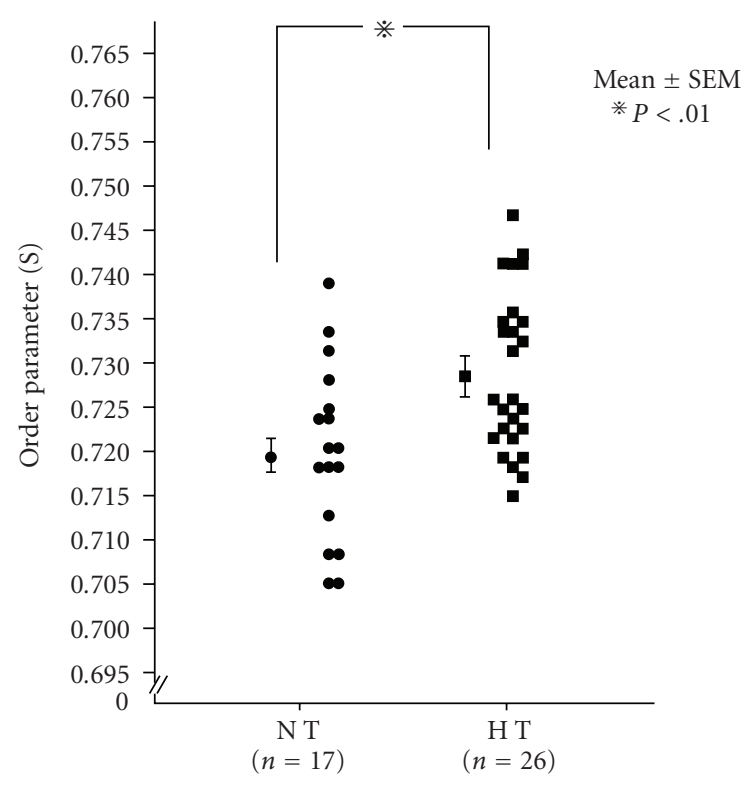

Figure 2: Membrane fluidity of red blood cells (RBCs) in hypertensive and normotensive men. The greater the value of the order parameter $(S)$ was, the lower the membrane fluidity of RBCs was.

stearate in the ESR spectra of RBC membranes was significantly higher in hypertensive men (HT) than in normotensive men (NT) (HT $0.728 \pm 0.002$, mean \pm SEM, $n=26$, NT $0.719 \pm 0.002, n=17, P<.01$ ) (Figure 2). The finding indicated that membrane fluidity of RBCs was decreased in hypertensive men compared with normotensive men.
3.2. Plasma Adiponectin, Plasma 8-iso-PG F2 $\alpha$, and Plasma NO-Metabolite Levels in Hypertensive and Normotensive Men. Plasma adiponectin concentration was lower in hypertensive men than in normotensive men (HT 7.0 $\pm 0.3 \mu \mathrm{g} / \mathrm{mL}, n=26$, NT $8.3 \pm 0.4 \mu \mathrm{g} / \mathrm{mL}, n=17, P<.05)$. The plasma NOmetabolites were also lower in hypertensive men than in normotensive men (HT: $36.3 \pm 2.6 \mu \mathrm{mol} / \mathrm{L}, n=26$, NT: $54.6 \pm 5.0 \mu \mathrm{mol} / \mathrm{L}, n=17, P<.01)$. In contrast, the plasma 8-iso-PG F2 $\alpha$ levels were significantly higher in hypertensive men than in normotensive men (HT: $3.33 \pm 0.27 \mathrm{nmol} / \mathrm{L}$, $n=26$, NT: $2.32 \pm 0.18 \mathrm{nmol} / \mathrm{L}, n=17, P<.05)$. In the overall analysis of hypertensive and normotensive men, plasma adiponectin concentration was positively correlated with plasma NO-metabolites $(r=0.334, n=43, P<.05)$ (Figure 3). It was also clearly shown that the plasma 8-iso-PG F2 $\alpha$ levels were inversely correlated with plasma adiponectin $(r=-0.313, n=43, P<.05)$ (Figure 4), and plasma NOmetabolites $(r=-0.396, n=43, P<.01)$ (Figure 5).

3.3. Relationship among Membrane Fluidity of RBCs, Plasma Adiponectin, Plasma 8-iso-PG F2 $\alpha$, and NO-Metabolite Levels in Hypertensive and Normotensive Men. The order parameter $(S)$ of RBCs was inversely correlated with plasma adiponectin $(r=-0.405, n=43, P<.01)$ (Figure 6) and plasma NO metabolite levels $(r=-0.342, n=43, P<.05)$ (Figure 7), and positively correlated with plasma 8 -isoPG F2 $\alpha(r=0.318, n=43, P<.05)$ (Figure 8$)$. These results might suggest that the reduced membrane fluidity of RBCs might be associated with hypoadiponectinemia, endothelial dysfunction, and increased oxidative stress. In a multivariate regression analysis, both of adiponectin and 8-iso-PG F2 $\alpha$ 


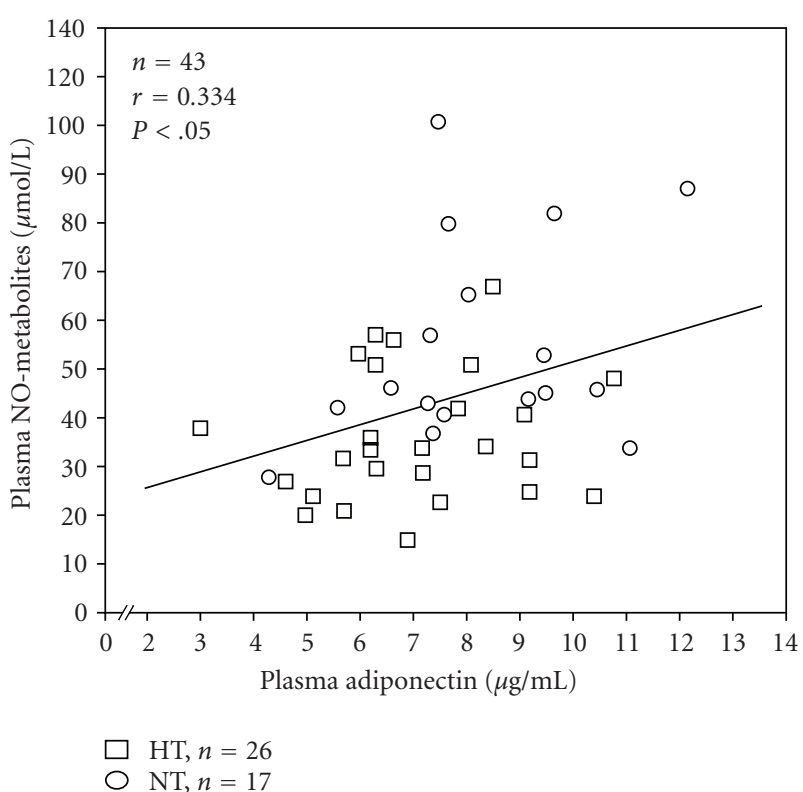

FIGURE 3: Correlation between plasma adiponectin and plasma nitric oxide (NO) metabolite levels in hypertensive and normotensive men.

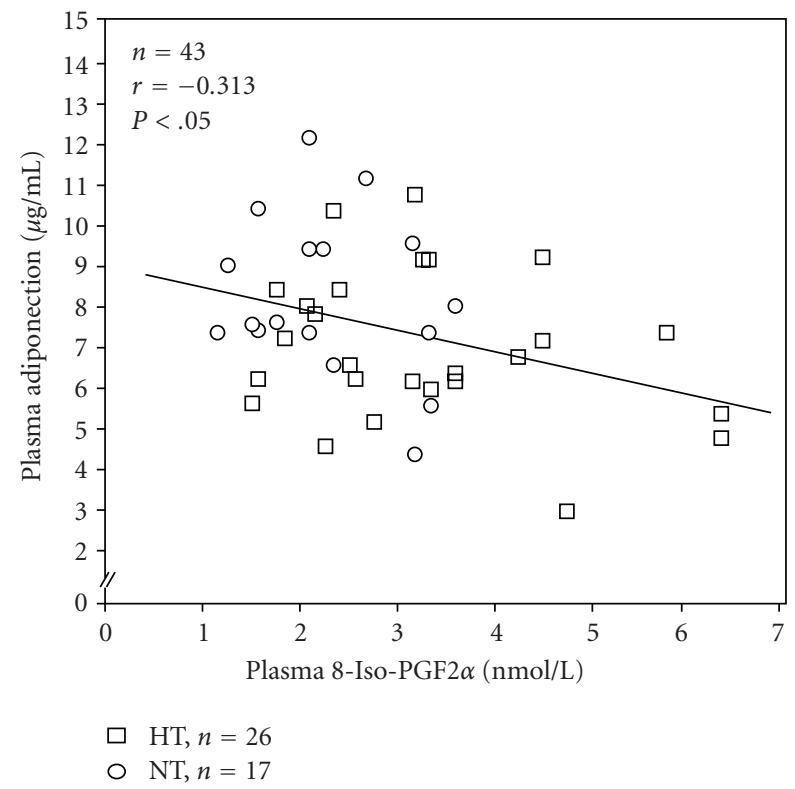

FIGURE 4: Inverse correlation between plasma 8-iso-PG F2 $\alpha$ and plasma adiponectin levels in hypertensive and normotensive men.

were significant determinants of membrane fluidity of RBCs after adjustment for general risk factors (Table 2).

\section{Discussion}

Recent studies have shown that both of adiponectin and oxidative stress might actively participate in the pathophysiology of obesity, hypertension, atherosclerosis, and

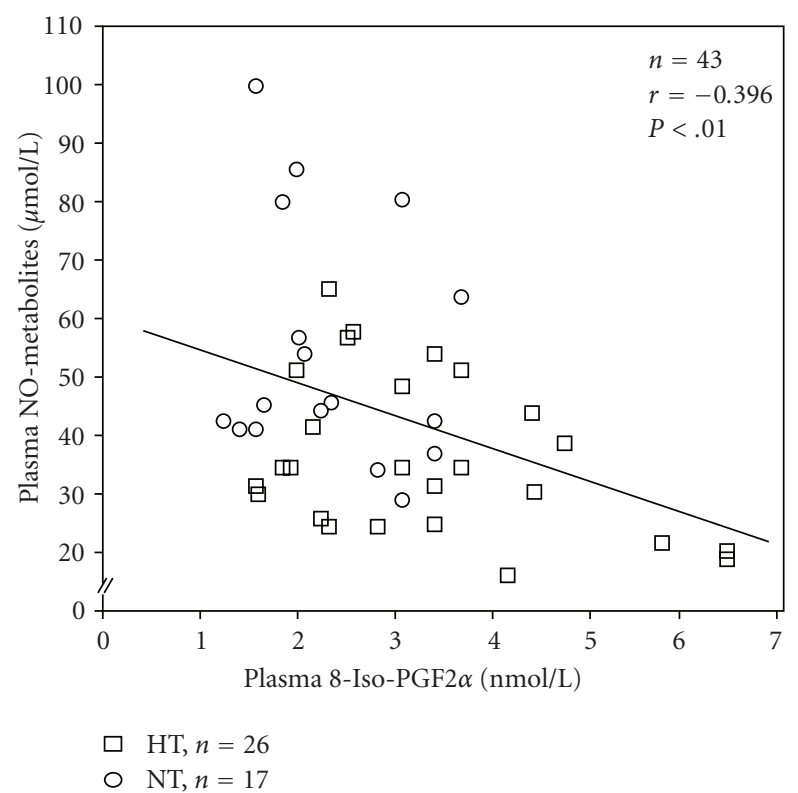

FIGURE 5: Inverse correlation between plasma 8-iso-PG F2 $\alpha$ and plasma nitric oxide (NO) metabolite levels in hypertensive and normotensive men.

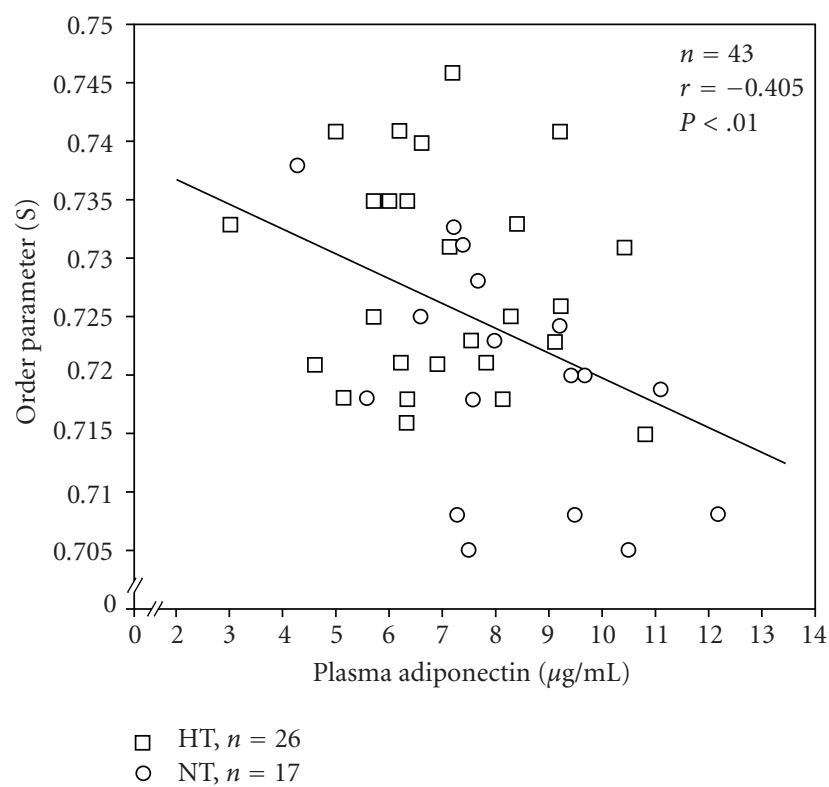

FIGURE 6: Inverse correlation between plasma adiponectin levels and the order parameter $(S)$ of red blood cells (RBCs) in hypertensive and normotensive men.

other cardiovascular and metabolic disease conditions [18]. The present study was performed to evaluate the possible relationship among adiponectin, oxidative stress, and membrane fluidity of RBCs in hypertensive and normotensive men using the ESR method. The results showed that the order parameter $(S)$ of RBC membranes in the ESR spectra was significantly higher in hypertensive men than in normotensive men, indicating that the membrane fluidity of 


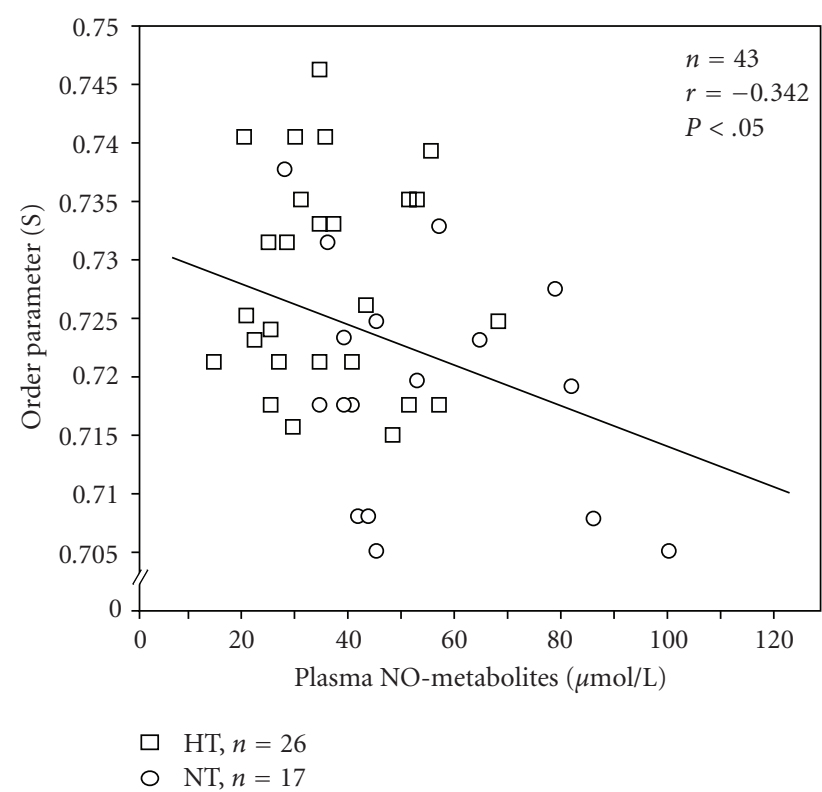

FiguRe 7: Inverse correlation between plasma nitric oxide (NO) metabolite levels and the order parameter $(S)$ of red blood cells (RBCs) in hypertensive and normotensive men.

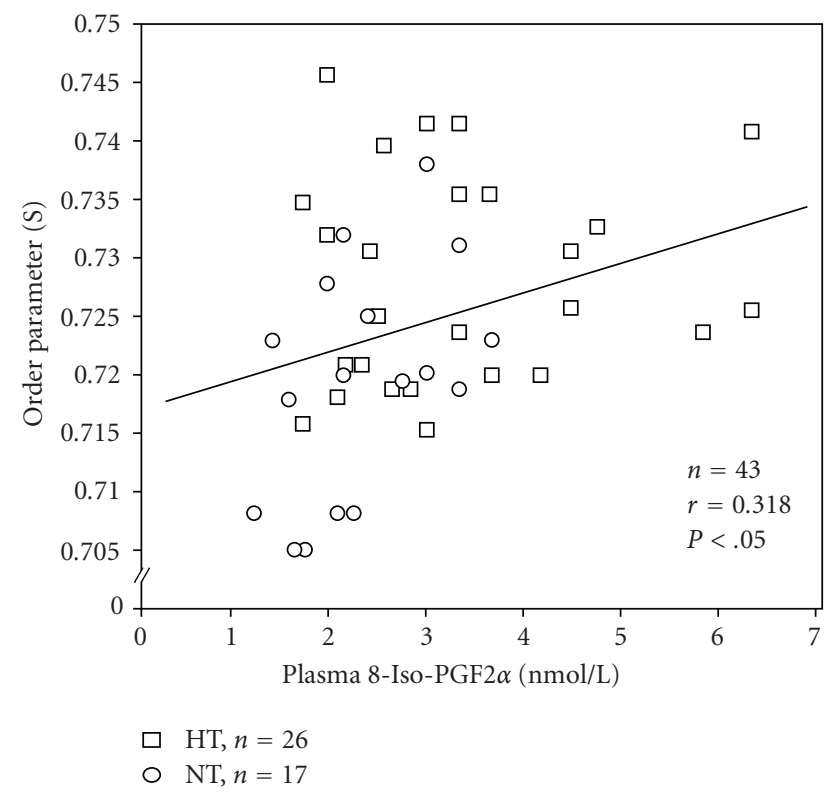

Figure 8: Correlation between plasma 8-iso-PG F2 $\alpha$ levels and the order parameter $(S)$ of red blood cells (RBCs) in hypertensive and normotensive men.

RBCs was decreased in hypertension. The finding might be consistent with our previous findings showing that the cell membranes were stiffer and less fluid in hypertension [2328].

In the present study, plasma adiponectin levels were significantly lower in hypertensive men than in normotensive men. In contrast, plasma 8-iso-PG F2 $\alpha$ levels (an index of oxidative stress) were significantly higher in hypertensive men than in normotensive men. Furthermore, it was shown that the order parameter $(S)$ of RBCs was inversely correlated with plasma adiponectin levels, and positively correlated with plasma 8 -iso-PG F $2 \alpha$ levels, indicating that the reduced membrane fluidity of RBCs was associated with hypoadiponectinemia and increased oxidative stress. Multivariate regression analysis also demonstrated that both adiponectin and plasma 8 -iso-PG F2 $\alpha$ were independent determinants of membrane fluidity of RBCs after adjustment for general risk factors. Because the deformability of RBCs might be highly dependent on the membrane fluidity $[21,22]$, the reduction in membrane fluidity of RBCs could cause a disturbance in the blood rheologic behavior and the microcirculation in hypertension.

Le Quan Sang et al. [32] demonstrated that shear rate, shear stress, and blood viscosity were correlated with membrane fluidity of RBCs. They proposed that in vivo shear forces might participate in the control of RBC membrane fluidity, and that RBCs might adapt their membrane properties to blood flow conditions [32]. Saldanha et al. [33] examined the RBC membrane fluidity of acute myocardial infarction, and showed that RBC membranes might become more rigid after myocardial infarction, which could contribute to the decreased RBC deformability and the increased blood viscosity in this group of patients. Cazzola et al. [34] also reported that membrane fluidity of RBCs was decreased in the obese subjects, and that RBC membranes in obese subjects had higher susceptibility to peroxidation. They proposed that a decrease in RBC membrane fluidity could contribute to a reduction of the rate of blood flow and the oxygen diffusion through the RBC membrane and its exchange with tissues. It might be possible that alterations in RBC membrane fluidity with elevated oxidative stress would be strongly linked to the progression of obesity and cardiovascular diseases.

Recently, it was demonstrated that adiponectin may stimulate production of $\mathrm{NO}$ in vascular endothelial cells in vitro [16]. In addition, it has been shown that plasma adiponectin was correlated with endothelium-dependent vasodilation of the brachial artery, suggesting that plasma adiponectin may be a useful marker of endothelial function in hypertensive subjects $[2,17]$. The present study demonstrated that the plasma levels of the NO metabolites were significantly lower in hypertensive men than in normotensive men. Furthermore, we showed that the plasma adiponectin levels were correlated with plasma $\mathrm{NO}$ metabolites in the overall analysis of normotensive and hypertensive men. It is, therefore, strongly suggested that hypoadiponectinemia could be associated with the reduced NO production and endothelial dysfunction. In an in vitro study presented earlier, we showed that NO significantly improved membrane fluidity of RBCs in hypertensive subjects [30]. The finding might propose that NO could have a crucial role in the regulation of membrane fluidity of RBCs, and further support the hypothesis that adiponectin might be associated with alterations in membrane fluidity of RBCs, at least in part, via the NO-dependent mechanism. However, the influence of adiponectin on the membrane lipid-protein interactions $[35,36]$ cannot be fully excluded. 
TABLE 2: Multivariate regression analysis for predicting order parameter $(S)$ of RBCs.

\begin{tabular}{lccc}
\hline & SRC & $t$-value & $P$ value \\
\hline Age & -0.025 & -0.142 & .8878 \\
Body mass index & -0.101 & -0.616 & .5417 \\
Fasting plasma glucose & 0.283 & 2.016 & .0515 \\
Systolic blood pressure & 0.201 & 1.390 & .1733 \\
Total cholesterol & -0.356 & -1.939 & .0607 \\
Plasma 8-iso-PG F2 $\alpha$ & 0.332 & 2.133 & .0400 \\
Plasma adiponectin & -0.322 & -2.195 & .0349 \\
\hline
\end{tabular}

$\mathrm{R}^{2}=0.389, n=43, F=3.186, P=.0102$.

SRC: standard regression coefficient.

The precise mechanisms by which oxidative stress could affect the membrane functions remain still unclear. Recently, it was shown that endothelium-dependent vasodilation was impaired in subjects with elevated oxidative stress levels $[18,19]$. The present study demonstrated that the plasma 8 -iso-PG F2 $\alpha$ levels were inversely correlated with plasma NO-metabolite concentration in the overall analysis of hypertensive and normotensive men. One hypothesis is that elevated oxidative stress could be associated with the reduced NO-production and endothelial dysfunction. In the present study, we demonstrated that the lower membrane fluidity of RBCs was associated with decreased plasma NO-metabolite levels. It is suggested that the effects of oxidative stress on membrane fluidity of RBCs might be mediated, at least in part, by reducing the NO-bioavailability, although direct actions of oxidative stress on membrane structural and functional properties cannot be excluded [37, 38].

Jubelin and Gierman [39] showed that RBCs of rats and humans are positive for NO synthase, which indicated that RBCs possess all the cellular machinery to synthesize their own NO. Chen and Mehta [40] provided direct evidence that human RBCs possess endothelium-type NO synthase in the cytosol. It would be possible that the membrane action of NO could be one of the mechanisms responsible for its beneficial effects in improving the rheological behavior of RBC membranes and the microcirculation. Further studies should be performed to assess more precisely the relationships among adiponectin, oxidative stress, NO, and membrane functions, and their contribution to the pathophysiology of hypertension.

Recently, it was shown that pharmacologic elevation of serum adiponectin with natural compounds derived from a medicinal herb, such as astragaloside II and isoastragaloside I, significantly ameliorated hyperglycemia, glucose intolerance, and insulin resistance in obese mice [41]. In addition, it was demonstrated that direct administration of adiponectin into the coronary artery reduced the myocardial infarction size and improved left ventricular function in pigs after ischemia-reperfusion injury [42]. These findings support the idea that adiponectin could be useful for the treatment of obesity and obesity-related cardiovascular disorders.

\section{Conclusion}

The results of the present study demonstrated that plasma adiponectin was significantly lower in hypertensive men than in normotensive men. In addition, plasma adiponectin concentration was positively correlated with plasma $\mathrm{NO}$ metabolite levels, and inversely correlated with plasma 8iso-PG F $2 \alpha$ levels. Furthermore, it was shown that the reduced membrane fluidity of RBCs was correlated with the decreased plasma adiponectin and $\mathrm{NO}$ metabolite levels and the increased plasma 8-iso-PG F2 $\alpha$ levels, suggesting that abnormalities in RBC membranes in hypertension might be associated with hypoadiponectinemia, endothelial dysfunction, and elevated oxidative stress. Although this is a cross-sectional and correlative study in Japanese men, the results of the present study could provide a hypothesis that both adiponectin and oxidative stress might have a close correlation with the rheologic behavior of RBCs and the microcirculation, and contribute to the pathophysiology of hypertension in men. Furthermore, it is possible that adiponectin could be a useful pharmacologic tool to improve membrane microviscosity in hypertension via the NOdependent mechanisms.

\section{Acknowledgments}

This study was supported in part by grants-in-aid for scientific research from the Ministry of Education, Science, Sports, Culture and Technology of Japan (15590604, 18590658, 20590710), the Daiwa Health Foundation (2001), the Uehara Memorial Foundation (2005), the Takeda Science Foundation (2006), the Salt Science Foundation (2007), and the Mitsui Foundation (2008).

\section{References}

[1] M. Kumada, S. Kihara, S. Sumitsuji et al., "Association of hypoadiponectinemia with coronary artery disease in men," Arteriosclerosis, Thrombosis, and Vascular Biology, vol. 23, no. 1, pp. 85-89, 2003. 
[2] N. Ouchi, M. Ohishi, S. Kihara et al., "Association of hypoadiponectinemia with impaired vasoreactivity," Hypertension, vol. 42, no. 3, pp. 231-234, 2003.

[3] M. Adamczak, A. Wiecek, T. Funahashi, J. Chudek, F. Kokot, and Y. Matsuzawa, "Decreased plasma adiponectin concentration in patients with essential hypertension," American Journal of Hypertension, vol. 16, no. 1, pp. 72-75, 2003.

[4] Y. Iwashima, T. Katsuya, K. Ishikawa et al., "Hypoadiponectinemia is an independent risk factor for hypertension," Hypertension, vol. 43, no. 6, pp. 1318-1323, 2004.

[5] M. Furuhashi, N. Ura, K. Higashiura et al., "Blockade of the renin-angiotensin system increases adiponectin concentrations in patients with essential hypertension," Hypertension, vol. 42, no. 1, pp. 76-81, 2003.

[6] A. Ceriello, "Possible role of oxidative stress in the pathogenesis of hypertension," Diabetes Care, vol. 31, supplement 2, pp. S181-184, 2008.

[7] A. A. Elesber, P. J. Best, R. J. Lennon et al., "Plasma 8-isoprostaglandin $\mathrm{F} 2 \alpha$, a marker of oxidative stress, is increased in patients with acute myocardial infarction," Free Radical Research, vol. 40, no. 4, pp. 385-391, 2006.

[8] R. Cangemi, F. Angelico, L. Loffredo et al., "Oxidative stressmediated arterial dysfunction in patients with metabolic syndrome: effect of ascorbic acid," Free Radical Biology and Medicine, vol. 43, no. 5, pp. 853-859, 2007.

[9] V. Dhawan and S. Jain, "Effect of garlic supplementation on oxidized low density lipoproteins and lipid peroxidation in patients of essential hypertension," Molecular and Cellular Biochemistry, vol. 266, no. 1-2, pp. 109-115, 2004.

[10] R. Rodrigo, H. Prat, W. Passalacqua, J. Araya, C. Guichard, and J. P. Bächler, "Relationship between oxidative stress and essential hypertension," Hypertension Research, vol. 30, no. 12, pp. 1159-1167, 2007.

[11] C. Vassalle, N. Botto, M. G. Andreassi, S. Berti, and A. Biagini, "Evidence for enhanced 8-isoprostane plasma levels, as index of oxidative stress in vivo, in patients with coronary artery disease," Coronary Artery Disease, vol. 14, no. 3, pp. 213-218, 2003.

[12] B. Wang, J. Pan, L. Wang, H. Zhu, R. Yu, and Y. Zou, "Associations of plasma 8-isoprostane levels with the presence and extent of coronary stenosis in patients with coronary artery disease," Atherosclerosis, vol. 184, no. 2, pp. 425-430, 2006.

[13] W. H. F. Sutherland, P. J. Manning, R. J. Walker, S. A. De Jong, A. R. Ryalls, and E. A. Berry, "Vitamin E supplementation and plasma 8-isoprostane and adiponectin in overweight subjects," Obesity, vol. 15, no. 2, pp. 386-391, 2007.

[14] J. A. Panza, A. A. Quyyumi, J. E. Brush Jr., and S. E. Epstein, "Abnormal endothelium-dependent vascular relaxation in patients with essential hypertension," New England Journal of Medicine, vol. 323, no. 1, pp. 22-27, 1990.

[15] K. Node, M. Kitakaze, H. Yoshikawa, H. Kosaka, and M. Hori, "Reduced plasma concentrations of nitrogen oxide in individuals with essential hypertension," Hypertension, vol. 30, no. 3 I, pp. 405-408, 1997.

[16] H. Chen, M. Montagnani, T. Funahashi, I. Shimomura, and M. J. Quon, "Adiponectin stimulates production of nitric oxide in vascular endothelial cells," Journal of Biological Chemistry, vol. 278, no. 45, pp. 45021-45026, 2003.

[17] K. C. B. Tan, A. Xu, W. S. Chow et al., "Hypoadiponectinemia is associated with impaired endothelium-dependent vasodilation," Journal of Clinical Endocrinology and Metabolism, vol. 89, no. 2, pp. 765-769, 2004.
[18] L. Loffredo, A. Marcoccia, P. Pignatelli et al., "Oxidative-stressmediated arterial dysfunction in patients with peripheral arterial disease," European Heart Journal, vol. 28, no. 5, pp. 608-612, 2007.

[19] M. Annuk, M. Zilmer, L. Lind, T. Linde, and B. Fellström, "Oxidative stress and endothelial function in chronic renal failure," Journal of the American Society of Nephrology, vol. 12, no. 12, pp. 2747-2752, 2001.

[20] Y. Yamori, Y. Nara, R. Horie, and A. Ooshima, "Abnormal membrane characteristics of erythrocytes in rat models and men with predisposition to stroke," Clinical and Experimental Hypertension, vol. 2, no. 6, pp. 1009-1021, 1980.

[21] K. Tsuda and I. Nishio, "Membrane fluidity and hypertension," American Journal of Hypertension, vol. 16, no. 3, pp. 259261, 2003.

[22] J. Zicha, J. Kuneš, and M.-A. Devynck, "Abnormalities of membrane function and lipid metabolism in hypertension," American Journal of Hypertension, vol. 12, no. 3, pp. 315-331, 1999.

[23] K. Tsuda, H. Iwahashi, and Y. Minatogawa, "Electron spin resonance studies of erythrocytes from spontaneously hypertensive rats and humans with essential hypertension," Hypertension, vol. 9, no. 6, pp. 19-24, 1987.

[24] K. Tsuda, Y. Kinoshita, K. Kimura, I. Nishio, and Y. Masuyama, "Electron paramagnetic resonance investigation on modulatory effect of $17 \beta$-estradiol on membrane fluidity of erythrocytes in postmenopausal women," Arteriosclerosis, Thrombosis, and Vascular Biology, vol. 21, no. 8, pp. 1306-1312, 2001.

[25] K. Tsuda and I. Nishio, "A selective estrogen receptor modulator, tamoxifen, and membrane fluidity of erythrocytes in normotensive and hypertensive postmenopausal women: an electron paramagnetic resonance investigation," American Journal of Hypertension, vol. 18, no. 8, pp. 1067-1076, 2005.

[26] K. Tsuda, "Electron paramagnetic resonance investigation on modulatory effect of benidipine on membrane fluidity of erythrocytes in essential hypertension," Heart and Vessels, vol. 23, no. 2, pp. 134-139, 2008.

[27] K. Tsuda, "Adiponectin and membrane fluidity of erythrocytes in normotensive and hypertensive men," Obesity, vol. 14, no. 9, pp. 1505-1510, 2006.

[28] K. Tsuda, "Oxidative stress and membrane fluidity of red blood cells in hypertensive and normotensive men: an electron Spin resonance investigation," International Heart Journal, vol. 51, no. 2, pp. 121-124, 2010.

[29] P. Muriel and G. Sandoval, "Nitric oxide and peroxynitrite anion modulate liver plasma membrane fluidity and $\mathrm{Na}^{+} / \mathrm{K}^{+}$ATPase activity," Nitric Oxide, vol. 4, no. 4, pp. 333-342, 2000.

[30] K. Tsuda, K. Kimura, I. Nishio, and Y. Masuyama, "Nitric oxide improves membrane fluidity of erythrocytes in essential hypertension: an electron paramagnetic resonance investigation," Biochemical and Biophysical Research Communications, vol. 275, no. 3, pp. 946-954, 2000.

[31] K. Tsuda, Y. Kinoshita-Shimamoto, K. Kimura, and I. Nishio, "Nitric oxide is a determinant of membrane fluidity of erythrocytes in postmenopausal women: an electron paramagnetic resonance investigation," American Journal of Hypertension, vol. 16, no. 3, pp. 244-248, 2003.

[32] K.-H. Le Quan Sang, J. Levenson, M. Del Pino, A. Simon, and M.-A. Devynck, "In vivo shear flow and erythrocyte membrane fluidity in hypertensive patients," British Journal of Clinical Pharmacology, vol. 36, no. 5, pp. 437-443, 1993.

[33] C. Saldanha, L. Sargenter, J. Monteiro, C. Perdigão, C. Ribeiro, and J. Martins-Silva, "Impairment of the erythrocyte 
membrane fluidity in survivors of acute myocardial infarction. A prospective study," Clinical Hemorheology and Microcirculation, vol. 20, no. 2, pp. 111-116, 1999.

[34] R. Cazzola, M. Rondanelli, S. Russo-Volpe, E. Ferrari, and B. Cestaro, "Decreased membrane fluidity and altered susceptibility to peroxidation and lipid composition in overweight and obese female erythrocytes," Journal of Lipid Research, vol. 45, no. 10, pp. 1846-1851, 2004.

[35] A. G. Lee, "Lipid-protein interactions in biological membranes: a structural perspective," Biochimica et Biophysica Acta, vol. 1612, no. 1, pp. 1-40, 2003.

[36] P. K. J. Kinnunen, A. Kõiv, J. Y. A. Lehtonen, M. Rytömaa, and P. Mustonen, "Lipid dynamics and peripheral interactions of proteins with membrane surfaces," Chemistry and Physics of Lipids, vol. 73, no. 1-2, pp. 181-207, 1994.

[37] C.-G. Zou, N. S. Agar, and G. L. Jones, "Oxidative insult to human red blood cells induced by free radical initiator AAPH and its inhibition by a commercial antioxidant mixture," Life Sciences, vol. 69, no. 1, pp. 75-86, 2001.

[38] F. Lenfant, J. J. Lahet, C. Vergely, F. Volot, M. Freysz, and L. Rochette, "Lidocaine inhibits potassium efflux and hemolysis in erythrocytes during oxidative stress in vitro," General Pharmacology, vol. 34, no. 3, pp. 193-199, 2000.

[39] B. C. Jubelin and J. L. Gierman, "Erythrocyte may synthesize their own nitric oxide," American Journal of Hypertension, vol. 9, no. 12 I, pp. 1214-1219, 1996.

[40] L. Y. Chen and J. L. Mehta, "Evidence for the presence of L-arginine-nitric oxide pathway in human red blood cells: relevance in the effects of red blood cells on platelet function," Journal of Cardiovascular Pharmacology, vol. 32, no. 1, pp. 5761, 1998.

[41] A. Xu, H. Wang, R. L. C. Hoo et al., "Selective elevation of adiponectin production by the natural compounds derived from a medicinal herb alleviates insulin resistance and glucose intolerance in obese mice," Endocrinology, vol. 150, no. 2, pp. 625-633, 2009.

[42] K. Kondo, R. Shibata, K. Unno et al., "Impact of a single intracoronary administration of adiponectin on myocardial ischemia/reperfusion injury in a pig model," Circulation: Cardiovascular Interventions, vol. 3, no. 2, pp. 166-173, 2010. 


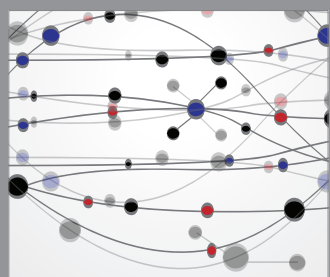

The Scientific World Journal
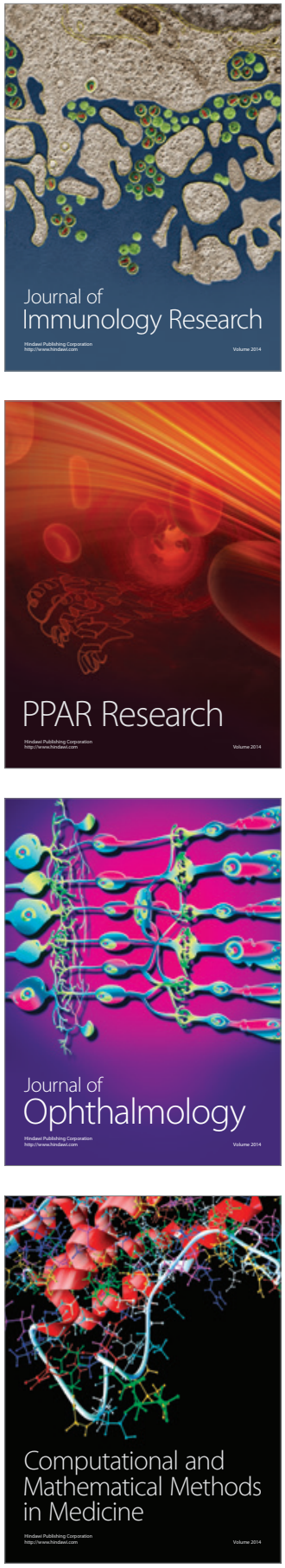

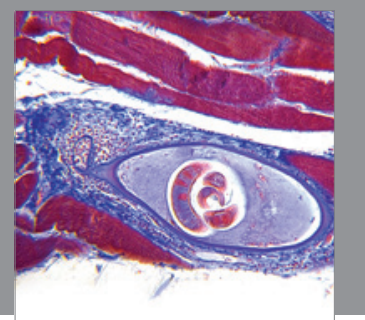

Gastroenterology

Research and Practice
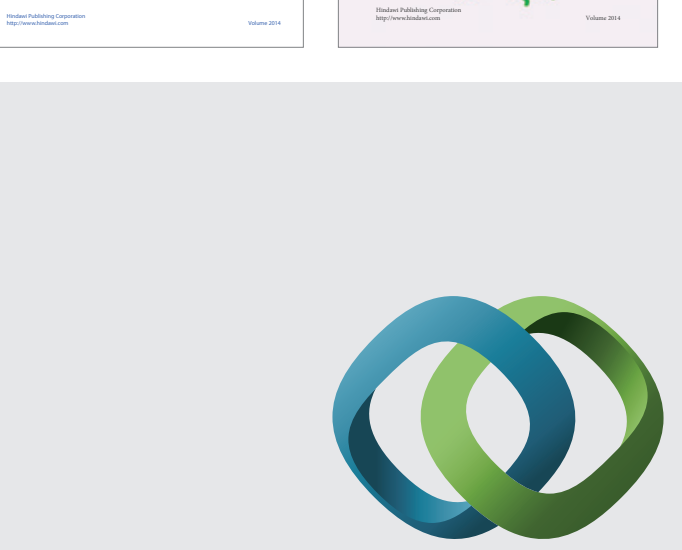

\section{Hindawi}

Submit your manuscripts at

http://www.hindawi.com
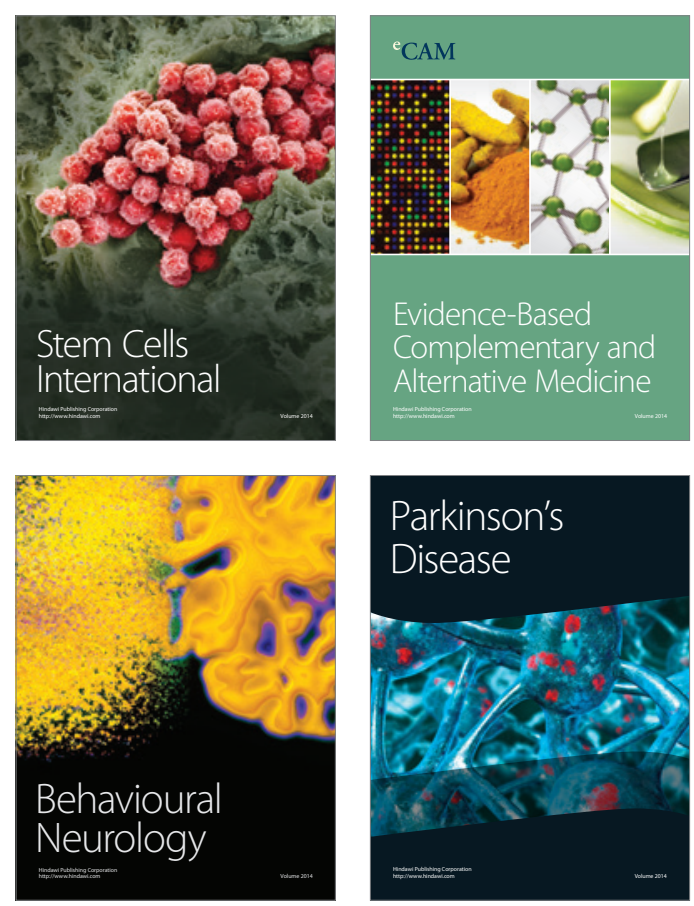

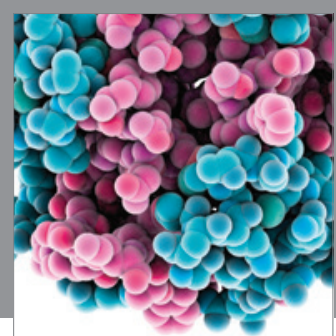

Journal of
Diabetes Research

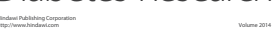

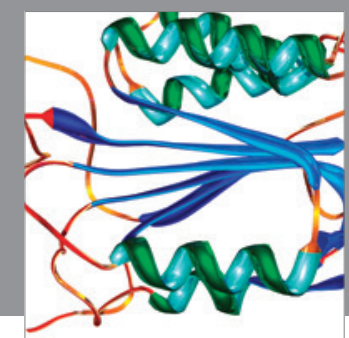

Disease Markers
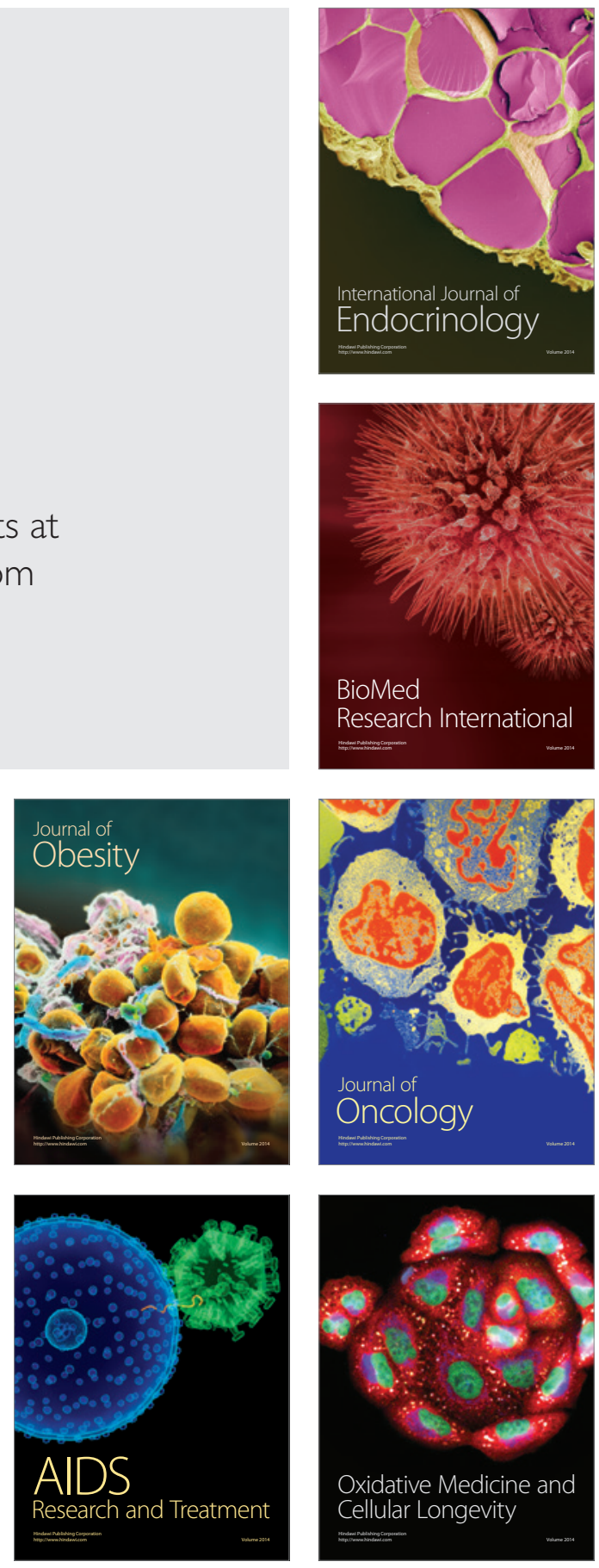